\title{
Un acercamiento hacia la apropiación y consumo de Instagram por parte de jóvenes universitarios
}

\section{An Approach Towards the Appropriation and Consumption of Instagram by University Students}

DOI 10.15517/rr.v99i2.39543

Laura Montero Corrales ${ }^{1}$

\begin{abstract}
${ }^{1}$ Escuela de Ciencias de la Comunicación Colectiva / Centro de Investigación de la Comunicación (CICOM), Universidad de Costa Rica, laura.monterocorrales@ucr.ac.cr

ORCID https://orcid.org/0000-0002-8973-0354
\end{abstract}

Fecha de recepción: 5 de noviembre del 2019 Fecha de aceptación: 21 de setiembre del 2020

\section{Resumen}

\section{Introducción}

El estudio de las redes sociales entre los jóvenes se ha convertido en una línea de investigación en crecimiento. Aunque existen numerosas investigaciones al respecto, el estudio de la apropiación y consumo social de la tecnología nos permite determinar cómo los jóvenes utilizan plataformas como Instagram y las incorporan de distintas formas a su vida diaria.

Objetivo

Por ello esta investigación tiene como objetivo descubrir y examinar las prácticas de apropiación y consumo de Instagram por parte de los estudiantes universitarios.

Método

El presente artículo presenta los resultados de un estudio en el cual se aplicó un cuestionario a 210 estudiantes, con edades entre 18 y 24 años de la Facultad de Ciencias Sociales de la Universidad de Costa Rica.

\section{Resultados}

Los resultados indican que los estudiantes universitarios utilizan esta red social como herramienta de interacción social, documentación, expresión personal y mirar lo que los otros hacen, así como un espacio de interacción con usuarios, marcas e influencers.

\section{Conclusiones}

Según los datos existen una serie de formas y prácticas por medio de las cuales los jóvenes universitarios hacen uso de Instagram, en especial su interés por usuarios de tipo influencers, que permiten comprender sus procesos de socialización y consumo de información a través de redes sociales."

Palabras clave: Redes sociales, Instagram, Jóvenes universitarios, Millennials, Publicidad. 


\section{Abstract}

\section{Introduction}

The study of social networks among young people has become a growing line of research. Although there are numerous investigations in this regard, the study of the appropriation and social consumption of technology allows us to determine how young people use platforms such as Instagram and incorporate them into their daily lives.

Objectiv

Therefore, this research aims to discover and examine the practices of appropriation and consumption of Instagram by university students.

\section{Method}

This article presents the results of a study that applied a questionnaire to 210 students of the Faculty of Social Sciences of the University of Costa Rica within the age range of 18 to 24 years old.

\section{Results}

The results indicate that university students use this social network as a tool for social interaction, documentation, personal expression and to look at what others do, as well as a space of interaction with users, brands and influencers.

\section{Conclusions}

According to the data, there is a variety of ways and practices through which young university students use Instagram, especially their interest in influencers, that allow to understand their processes of socialization and consumption of information through social networks.

Key words: Social network, Instagram, College students, Millennials, Advertising.

\section{Uma abordagem para a apropriação e consumo de Instagram por jovens universitários}

\section{Resumo}

\section{Introdução}

$\mathrm{O}$ estudo das redes sociais entre os jovens tem se tornado uma linha de pesquisa crescente. Embora existam inúmeras investigações a este respeito, o estudo da apropriação e do consumo social da tecnologia permite-nos verificar como os jovens utilizam plataformas como o Instagram e como as incorporam de diferentes formas no seu quotidiano.

Objetivo

Portanto, esta pesquisa tem como objetivo conhecer e examinar as práticas de apropriação e consumo do Instagram por estudantes universitários.

\section{Método}

Este artigo apresenta os resultados de um estudo no qual foi aplicado um questionário a 210 alunos, com idades entre 18 e 24 anos, da Faculdade de Ciências Sociais da Universidade da Costa Rica.

\section{Resultados}

Os resultados indicam que os universitários utilizam essa rede social como ferramenta de interação social, documentação, expressão pessoal e observação do que os outros fazem, além de espaço de interação com usuários, marcas e influencers. 


\section{Conclusões}

De acordo com os dados, há uma série de formas e práticas pelas quais jovens universitários fazem uso do Instagram e principalmente com usuários do tipo influencers que lhes permitem compreender processos de socialização e consumo de informação por meio das redes sociais.

Palavras-chave: Redes sociais, Instagram, Jovens universitários, Millennials, Publicidade.

\section{Introducción}

\section{Apropiación y uso de la tecnología}

Estamos viviendo una época inmersa en un proceso de digitalización creciente. Así lo establece Andrés (2012) en «Prácticas culturales en red: reflexión sobre la participación y producción de los internautas», en el cual explica que el uso expandido de computadoras y teléfonos móviles con conexión a Internet ya forma parte de la vida cotidiana y transforman, no solo las formas de comunicación, sino también las instancias de socialización, el funcionamiento de la economía, las prácticas pedagógicas y los consumos culturales. Este fenómeno ha generado un impacto profundo tanto en las prácticas humanas como las relaciones interpersonales, además del impacto en las prácticas de consumo y relaciones con los medios gracias a una serie de herramientas como las redes sociales, específicamente en Instagram.

Maestri (2011), en su investigación sobre audiencias digitales, sostiene que la transportabilidad, la conectividad permanente, la miniaturización y el fácil manejo de la gran mayoría de los dispositivos tecnológico, provocan que las posibilidades de interacción, de conexión y de creación aumenten en relación con los dispositivos comunicacionales anteriores. Esto también plantea una infinidad de prácticas, acciones y usos que cada sujeto va generando y cambiando según sus propias necesidades o afinidades con las tecnologías a su alcance; por lo que se hace difícil hablar de una práctica promedio en relación al uso de las tecnologías y los medios de comunicación e información, tal como se pensaba en relación a las prácticas de las audiencias masivas.

Sobre esto, Andrés (2012) explica que este crecimiento constante del uso de artefactos que navegan en Internet, ha modificado la forma de comunicarse de las personas y las posibilidades de acceso y difusión de información y datos. La digitalización de contenido constituye una transformación en la creación artística y en los modelos de negocio de las industrias del entretenimiento. Y también presenta un rasgo novedoso: le otorga - potencialmente - a cada usuario la posibilidad de crear y difundir sus producciones y materiales personales, o modificar los existentes, con el fin de otorgarles otro sentido.

En este caso, siguiendo a Maestri (2011), usamos la noción de consumo para referirnos a la producción. Es decir, se entiende que el acto de consumir supone otras acciones relacionadas, como opinar, comentar, modificar, intervenir, etiquetar y participar, en definitiva interactuar. De la misma forma, la persona autora afirma que el consumo puede darse de forma asincrónica (cuando se graba o se guarda el contenido para ser visto cuando la persona usuaria quiera); o 
de forma sincrónica en modo móvil (por medio de teléfonos celulares o tabletas): donde es posible producir en el momento que la persona usuaria lo desee.

Sobre esta práctica de producción y consumo y con el objetivo de identificar posibles prácticas de apropiación por parte de los jóvenes en Instagram, tomamos como referencia el trabajo de Michel de Certeau ${ }^{1}$. El autor se interesa por la práctica del hombre común, sus trucos para gestionar situaciones cotidianas, pero específicamente sobre el estudio del uso que hacen los grupos o individuos de los objetos como la televisión, que como el autor menciona no sólo debería tratar sobre el análisis de las imágenes o cuánto tiempo está frente al televisor, sino complementarse con el estudio de lo que el consumidor cultural fabrica durante estas horas y con estas imágenes (de Certeau 1990):

Dicho de otro modo, debe haber una lógica de estas prácticas. Es regresar al problema, ya antiguo, de lo que es un arte o una «manera de hacer». De los griegos a Durkheim, pasando por Kant, una larga tradición se ha dedicado a precisar las formalidades complejas (y para nada simples opobres") que pueden dar cuenta de aquellas operaciones. A través de este sesgo, la «cultura popular» se presenta de un modo diferente, así como toda una literatura llamada «popular»: se formula esencialmente en «artes de hacer» esto o aquello, es decir, en consumos combinatorios y utilitarios. Estas prácticas ponen en juego una ratio «popular», una manera de pensar investida de una manera de actuar; un arte de combinar indisociable de un arte de utilizar. (de Certeau 1990, 37).

En esencia, de Certeau se interesa en el reconocimiento de las «acciones» que tienen una formalidad y un uso, y que organizan discretamente el trabajo del consumo de un objeto o una práctica dada (de Certeau 1990). Desde este punto de vista, la apropiación de una tecnología, por parte de quien la usa, estaría definida en términos de los nuevos proyectos de uso que emergen en el seno de su vida cotidiana. Para esto, el autor establece que el análisis de las prácticas cotidianas de las personas consumidoras, se puede ordenar en tres niveles: 1) las modalidades de la acción, 2) las formalidades de las prácticas y 3) los tipos de operación especificados por las maneras de hacer. Estas últimas constituyen las prácticas mediante las cuales los usuarios se reapropian del espacio organizado de la producción cultural, y son las que corresponden a los usos ideados por agentes externos a los usuarios que se denominan como usos estratégicos o predeterminados y los usos que derivan.

Para Manovich (2008) la relación entre estrategias y tácticas se han reconfigurado según el análisis hecho por Certeau en 1980. Para Manovich (2008), desde 1980 el consumidor y las industrias culturales han convertido cada subcultura, como la bohemia o los punks, en productos. De esta forma, las tácticas culturales de las personas quienes las practicaban fueron

\footnotetext{
${ }^{1}$ Se cuenta lo que es utilizado, no las maneras de utilizarlo. Paradójicamente, éstas se vuelven invisibles en el universo de la codificación y de la transparencia generalizadas. De estas aguas que se insinúan por todas partes sólo son perceptibles los efectos (la cantidad y la localización de los productos consumidos). Circulan sin ser vistas, reconocibles solamente en los objetos que desplazan y hacen desaparecer. Las prácticas del consumo son los fantasmas de la sociedad que lleva su nombre. Como los «espíritus» de antaño, constituyen el principio multiforme y oculto de la actividad productora (de Certeau 1990, 42).
} 
convertidas en estrategias y vendidas a quienes no pertenecían a la subcultura, es decir quienes no la practicaban. Ahora las personas tienen acceso a muchos estilos de vida, subculturas que se caracterizan por el estilo de música o de ropa a los cuales se pueden acceder. En resumen, Manovich (2008) indica que las estrategias actuales para redes sociales, se parecen más en las tácticas originalmente formuladas por de Certeau, ya que a las empresas, que desarrollan contenidos para redes sociales, les interesa tener la mayor cantidad de visitas posibles y una gran cantidad de personas usuarias dentro de la comunidad. Por lo que su interés se centra en que, quienes las usan, compartan información sobre sus vidas en estas plataformas.

Es por esto que, para el estudio del consumo social de estas tecnologías, nos aprovechamos de la Teoría de la Apropiación de Serge Proulx, (citado por Siles 2004) quien la define como el «dominio técnico y cognitivo del artefacto» (Siles 2004, 26), y plantea que la apropiación social de la tecnología se genera cuando se integra el objeto técnico en la práctica cotidiana del usuario y crea acciones encaminadas a generar novedad en la práctica social e impactar finalmente en un proceso de innovación Esta Teoría de la Apropiación permite determinar cómo utilizan los usuarios estos canales de comunicación y por qué «la perspectiva de estudio de la apropiación se centra en los modos de personalizar y "hacer propia" una determinada tecnología por medio de su uso. En este sentido, la apropiación vendra a estudiar las formas por medio de las cuales el usuario hace suya la tecnología y la incorpora creativamente al conjunto de sus actividades cotidianas» (Siles 2004, 255).

Acerca del tema, Proulx (citado por Siles 2004) define algunas preguntas que sirven para enriquecer el alcance de las investigaciones de la apropiación y el consumo: «¿Qué nuevas dinámicas surgen por la apropiación de las mismas (i. e. lenguajes, convenciones de uso, prácticas, comportamientos)? ¿Qué tipo de diferencias concretas de apropiación de un objeto o producto tecnológico existen según el género del usuario o según su edad?» (Siles 2004, 80).

Burgess (2006) indica que ha habido un incremento notable en la participación de los usuarios en esta cultura de los medios. Nuevas dinámicas de apropiación han aparecido por el crecimiento repentino de los blogs y el empoderamiento que brindan estas tecnologías digitales, que permiten el uso por parte de personas comunes y usuarios más activos que influyen sobre otros usuarios. Esta es una combinación que solo las redes sociales, como medios de comunicación, permiten a las personas, en las cuales cualquier seguidor de una página puede participar de las conversaciones, tanto como consumidor como productor. Así lo establece Manovich (2008), quien indica que, en el 2007, solo entre el $0.5 \%$ y $1.5 \%$ de los usuarios de sitios como Youtube o Wikipedia, crearon su propio contenido.

En este sentido, siguiendo a de Certeau, el acercamiento a los procesos de apropiación consistiría en detectar los mecanismos por los cuales los individuos logran la capacidad de independencia para llevar a cabo una serie de prácticas en la vida cotidiana. Pero también logran conocer sobre las distintas herramientas que tienen a su alcance, así como la comprensión de cómo se usan, logrando satisfacer sus necesidades e intereses. Por ejemplo, si se quisiera estudiar el proceso de apropiación de Internet, el mismo no debería limitarse a las tareas o pasos de cómo utilizar Internet, sino que debería enfocarse desde una serie de situaciones de vida de 
las personas, en cómo utilizan esa herramienta. Este estudio busca lograr un acercamiento hacia la apropiación y consumo de Instagram por parte de jóvenes universitarios considerados millennials desde el campo del mercadeo.

\section{Millennials}

De manera tal que podríamos indicar que los millennials son aquellos individuos que tienen entre 18 y 35 años y nacieron antes del cambio de milenio. Tomando como referencia la teoría de Kotler y Keller (2012), pioneros del marketing moderno, se dice que los millennials han sido mundialmente identificados como las personas nacidas entre 1980 y 1996. Desde el campo del mercadeo, es una práctica común que los mercadólogos clasifiquen a los consumidores en categorías para poder segmentar sus gustos y preferencias, de manera que sea más efectivo comunicar los mensajes relacionados a los bienes y servicios que las empresas producen. Sin embargo, en este período se presentan tantas variaciones como las teorías y estudios sobre su comportamiento. En consecuencia, los millennials constituyen la futura raíz de consumidores y usuarios dentro de un mercado definido por un conjunto de características, necesidades y demandas totalmente nuevas. Como consecuencia, se han convertido en el blanco de toda acción publicitaria y del mercadeo, ya que conforman uno de los grupos más grandes en población, gracias a su capacidad económica y estilo de vida.

Específicamente en Costa Rica, según el estudio «Costa Rica cuenta con su propio estudio de generaciones» (GENTICO: La verdad sobre las Generaciones en Costa Rica. (UNIMER y Kölbi, 2016), realizado por Unimer y la marca Kölbi, el cual contempló entrevistas con expertos, entrevistas de corte etnográfico y 1.802 entrevistas cara a cara con costarricenses de 12 a 80 años, tomamos la siguiente clasificación como partida para la investigación: existe una «generación satelital» (la cual incluye al costarricense que representa un cambio; alguien que recibe algunas señales del pasado, pero ya conectado con la globalización y retransmite otros valores a las siguientes generaciones; son personas nacidas entre 1961-1981 y que hoy en día tienen entre los 37 y 57 años de edad); y hay una «generación digital» (la cual describe que su principal medio de comunicación son las redes sociales e Internet, que son los nacidos en la era digital, entre 1982-1999 y que hoy en día tienen entre los 17 y 34 años de edad). De acuerdo con esto, los millennials entrarían en esta segunda categoría. De acuerdo con el estudio, las personas de esta generación «crecen viviendo el cambio tecnológico con la llegada de la computadora, el Internet y el acelerado avance de la telefonía celular, y es por eso que su curva de aprendizaje es acelerada y muestran pocas barreras hacia el uso de la tecnología.

Datos del 2016 del Instituto Nacional de Estadística y Censos (INEC) y el Centro Centroamericano de Población (CCP) de la Universidad de Costa Rica, en la Gran Área Metropolitana (que comprende a las provincias de San José, Heredia, Cartago y Alajuela), indican que los millennials costarricenses ya superan el millón de personas (1.019.234 personas 
a marzo de 2016) ${ }^{2}$. Esta cifra equivale a una quinta parte de la población de Costa Rica (20,84\%). A nivel latinoamericano se estima que el 30\% de la población calza en este grupo. A esto podemos sumar los datos del Tribunal Supremo de Elecciones de Costa Rica (TSE) a enero de 2017, donde se señalaba que el padrón electoral tenía un total de 3.256 .590 personas. De estas, 1.632.571 eran mujeres y 1.624.019 eran hombres. De acuerdo con el rango de edad, el $40 \%$ eran millennials, el $12 \%$ correspondía a adultos mayores y el $48 \%$ eran personas entre 35 y 64 años. Por esto creemos que el mundo digital ha vivido cambios que sin duda alguna lo han transformado drásticamente desde la irrupción de los millennials.

Esta transformación del ecosistema digital donde participan redes sociales, marcas y usuario, ha impactado la forma en que las empresas se comunican en redes sociales, ya que estos tipos de comportamientos por parte de los millennials, les permite a las marcas crear y diseñar estrategias a acordes a este público, redefiniendo la manera de comunicarse, a través de sus estrategias publicitarias.

Una característica que define en mayor medida estas personas es la hiperconectividad permanente a través de los smartphones ingresando diariamente a redes sociales como Instagram, donde buscan alimentar de forma constante una cualidad fundamental del ser humano, la sociabilidad, pero en el mundo digital. Rescatamos la reflexión de Manuel Castells (2011) sobre este tema donde indica que «La conexión que proporciona la Internet es tan fuerte que genera nuevas redes y comunidades sociales afines y que permiten desarrollar vínculos fuertes o débiles dependiendo de su interacción». Así, en las redes sociales actuales, podemos afirmar que vivimos la evolución del ser humano hacia un nuevo tipo de individuo «SIEMPRE CONECTADO», que no se parece, tanto en aspectos importantes de su proceso de socialización como en su desarrollo en general, a sus predecesores.

Un millennials siempre conectado, socializando, consumiendo y produciendo, en redes sociales como Instagram.

\section{Instagram}

La red social se define a sí misma como «una manera simple, divertida y creativa de capturar, editar y compartir fotos, videos y mensajes con amigos y familiares» (Instagram, s.f.). Actualmente posee 400 millones de personas usuarias - presenta un crecimiento explosivo desde su adquisición-, y el $73 \%$ de quienes la usan son una población que está entre los 15 y 35 años. Esto significa que su público está compuesto principalmente por personas adultas jóvenes. Según datos de la compañía, para abril del 2017, la aplicación contaba con 700 millones de personas usuarias (Instagram Press 26 de abril de 2017). En nuestro país Instagram cuenta con 1100000 personas registradas, de las cuales 510000 son hombres y 650000 mujeres (Instagram Press 26 de abril de 2017).

\footnotetext{
${ }^{2}$ Esta cifra equivale a una quinta parte de la población de Costa Rica (20,84\%). Aunque a nivel latinoamericano se estima que el $30 \%$ de la población calza en este grupo (https://semanariouniversidad.com/pais/millenials-lageneracion-las-pantallas-las-redes/)
} 
Específicamente, según el estudio «Red506 2017-VII Edición-El Financiero» (2017), esta es la tercera red social más utilizada, después de Facebook y WhatsApp. En este mismo estudio, las personas usuarias indicaron que la utilizan para compartir fotos con amistades y seguidores, así como seguir personalidades, marcas y empresas.

Asimismo, el estudio «Estadísticas de Instagram 2017 (América Latina)» (2017) recopiló datos durante el mes de mayo de 2017 en América Latina, los cuales indicaron que hay más de 60 millones de personas registradas en Instagram, de las cuales 28 millones son hombres y 32 millones mujeres aproximadamente. También se menciona que, con excepción de Guatemala, las mujeres superan por mucho a los hombres en cantidad de población registrada en Instagram.

Esta información se vio respaldada por el estudio de Pew Research, realizado en 2015 con residentes de los EE.UU., en el cual se descubrió que Instagram es más usado por mujeres que por hombres. El estudio mostró que $31 \%$ de las mujeres y $24 \%$ de los hombres usan Instagram regularmente para dar un «me gusta», compartir y publicar (Hootsuite 2017).

Dentro del estudio «An Examination of Users and the Commodification of Instagram», Macon $(2017,2)$ identificó que, dentro de la comunicación masiva que se da dentro de las diversas redes sociales, la principal práctica se da utilizando uno de estos dos modelos: el vertical o el horizontal. En el primero, una persona o corporación se comunica hacia las personas usuarias, basándose en la creación y distribución de contenido publicitario, pero nunca en la interacción. Tradicionalmente, este modelo se utilizaba en medios masivos como radio, televisión y periódicos, los cuales regían la distribución de poder comunicativo y las audiencias tenían una influencia relativamente baja en la modificación o solicitud de contenido. Por otro lado, el modelo horizontal se produce usualmente entre las personas usuarias y la diaria interacción regular.

Con la llegada del Internet, se popularizó el concepto de user generated content (UGC), es decir, contenido creado por quienes usan la red. Se pasa del modelo vertical al horizontal, en el cual las personas pueden decidir qué ver y a cuáles comunidades pertenecer. Como resultado, plataformas como Instagram funcionan como un nicho para las compañías, pues les permite identificar los intereses de sus audiencias y los usos que le dan a la red social.

Ahondando en lo anterior, un estudio realizado por Eunji, Jung-Ah, Jang Ho, and Yongjun (2015), arrojó que los motivos de uso de Instagram se pueden dividir en cinco principales:

- Social Interaction: para interactuar con personas, mantener una buena relación con los demás, crear redes, conocer las cosas que suceden a mi alrededor. Conectar y reunirse con personas que comparten intereses similares, estar informado sobre lo que hacen amigos y familiares, para así mantener comunicación con ellos.

- Archiving: para guardar eventos diarios a través de fotos, crear un espacio personal; grabar una un viaje a través de photomap, hacer blogs personales, para tomar fotos de lujo y guardarlas en línea; para actualizar fotos y videos con varios filtros aplicados.

- Self-expression: para actualizar contenidos, para ser notado por otros, para expresar lo que se es realmente, para compartir información personal con otros. 
- Escapism: escapar de la realidad, olvidarse de problemas, evitar la soledad, conseguir lo que se quiere sin mucho esfuerzo y relajarse.

- Peeking: para navegar por las fotos relacionadas con mis intereses, ver la vida de las celebridades, ver una variedad de fotos interesantes.

Algunos estudios en EE.UU. han mostrado que la popularidad de Instagram podría haber superado la de Facebook, especialmente entre personas universitarias (Endres 2013). La población estudiantil prefiere seguir marcas en redes sociales con el fin de ver los contenidos publicados por las empresas.

Un estudio realizado por Brandwatch Analytics (2016) mostró que, para el año 2016, el $48.8 \%$ de las marcas comerciales utilizaban esta red social y que, para el 2017, se esperaba que este porcentaje aumentara a un $70.7 \%$. Esto demuestra que las marcas consiguen llegar a las personas usuarias a través de esta plataforma, quienes estas están dispuestas a interactuar con las primeras. Junto con lo anterior, las tres principales razones por las que la mayoría de la población usuaria sigue a una marca en Instagram son las siguientes: el $62 \%$ admite hacerlo simplemente porque les encanta la marca, el 54\% dice seguir marcas para informarse sobre los últimos productos que ofrecen, y el $48 \%$ sigue los perfiles de marcas porque las publicaciones de estas les parecen interesantes o divertidas.

También, en el estudio de Carah (2016) se analizan las actividades, específicamente de la marca global Smirnoff Vodka y la marca de moda General Pants, en Instagram. El análisis examina una secuencia de 100 imágenes de cada marca, con los hashtags, en el caso de General Pants, \#generalpants y \#gpwetdream; en el caso de Smirnoff, \#doubleblackhouse y \#vipublic. En este estudio, se codificaron las imágenes para determinar quién las había creado (marca, consumidor o intermediario cultural), el género de cualquier cuerpo presente en las imágenes y el compromiso con las mismas, medidos por los «me gusta» y los comentarios. Encontraron que Instagram ilustra cómo el trabajo discursivo-simbólico y el guiado por datos están correlacionados.

Al crear contenido, también se tiene en mente aspectos técnicos como los algoritmos, los cuales pueden traer más atención a la comunicación de la marca en Instagram. El análisis anterior en relación al uso de hashtags reveló cómo se replican reglas de género en los posts y esto conlleva a que el algoritmo de Instagram haga lo mismo, al entender qué tipo de contenidos generar más atención por parte de los usuarios de manera natural y emular estos comportamientos en la decisión de qué contenidos son mostrados con mayor frecuencia a los usuarios.

Otro elemento que genera atención, según Bloomaert (2016) es el rol identificado de Instagram que permite a pares hablar y compartir información entre ellos, término conocido como Word of Mouth. Esto permite a las personas usuarias experimentar una conversación imparcial acerca de las marcas por parte de los Brand Loyals (usuarios fieles a la marca), generando dos resultados: por una parte, la Word of Mouth expone a la población usuaria o crea conciencia de marca de una manera auténtica. Por otra, quienes interactúan con los Brand Loyals tienden a sentirse obligados a ser también fieles a la marca, esto por querer pertenecer a 
un grupo específico. Según Macon (2017), en Instagram se acompaña el factor Word of Mouth con un lenguaje seleccionado para una comunidad online específica. Es decir, se construyen las características de cómo se ve una persona que venda un determinado producto. Es debido a esta personificación y la sensación interacción humana que las personas tienen dificultad al identificar publicaciones de Instagram de otro usuario como «publicidad».

Además de las publicaciones que se pueden hacer en el muro, Instagram cuenta con la característica Historias de Instagram, la cual permite establecer relaciones con usuarios influencers o personas que hablan de una marca, así como repostear o generar contenido. Debido a esto, los influencers publican historias a su comunidad de seguidores y tienen la posibilidad de utilizar hashtags y geolocalizarse para ser encontrados fácilmente por personas interesadas, así como potenciales o actuales clientes. Además, gracias a datos mesurables de interacción por quienes usan esta red, se puede comparar éxito de publicaciones, así como probar y crear contenido siguiendo información obtenida de estadísticas. En su actualización del 20 de junio de 2018, Instagram introdujo la modalidad de Instagram televisión o IGTV, dedicada a la creación y visualización de videos verticales. El periódico La Vanguardia recalca que esto puede ser una estrategia para romper con el esquema de videos horizontales que plataformas como Youtube han impuesto durante años. A diferencia de las Historias de Instagram, IGTV no tiene una limitación de un minuto y sus videos pueden durar hasta una hora. Sus «canales» son las mismas personas creadoras y cuenta con cuatro modalidades: «For You», «Following», «Popular» $\mathrm{y}$ «Continue Watching».

Es por esto que el presente artículo tiene como objetivo examinar las prácticas de apropiación y consumo que realizan las personas jóvenes universitarias en Instagram. La población considerada pertenece a la Facultad de Ciencias Sociales de la UCR, específicamente estudiantes de las carreras de Ciencias de la Comunicación Colectiva, Antropología, Psicología y Ciencias Políticas; entre los 18 y 24 años de edad. La información se obtuvo como resultado de un cuestionario online aplicado a 210 estudiantes, durante los meses de junio y julio del 2018. Interesó examinar cómo se comportaban estas personas usuarias en Instagram, describir su proceso de consumo de contenidos y definir cuáles son las acciones de interacción que llevan a cabo con los perfiles de marcas y líderes de opinión en esta red social.

\section{Investigación}

La aplicación del cuestionario permitió recoger la información necesaria para poder cuantificar y universalizar la información, y así obtener los datos de la mayor cantidad posible de millenials ${ }^{3}$. Gracias a la revisión bibliográfica, y mediante la recopilación de instrumentos publicados, se obtuvo un conjunto de preguntas y se clasificaron en función de las dimensiones de estudio. Posteriormente se diseñaron preguntas específicas y se construyó la primera versión del cuestionario.

\footnotetext{
${ }^{3}$ Enlace a instrumento https://bit.ly/3bAK4gm
} 
Se aplicó el cuestionario a 210 personas, 113 de corresponden a estudiantes de la carrera de Ciencias de la Comunicación Colectiva y 97 a las carreras de Antropología, Psicología y Ciencias Políticas. De estos, 190 aseguraron que sí utilizaban Instagram, por lo que este número corresponderá al 100\% dentro de estos resultados. Además, 138 personas se identificaron como mujeres, 49 como hombres y 3 como otro. La edad prominente dentro del cuestionario fue de 18 años, específicamente mujeres, con un 17\% (34 personas) y hombres, con un 8\% (16 personas). Seguidamente, el grupo con más uso fue las mujeres de 20 años con un $13 \%$ (25 personas). En cuanto a los hombres, tanto las edades de 19 como de 23 años obtuvieron el mismo porcentaje de uso Instagram, el cual fue de de 3\% (7 personas). En cuanto al tercer grupo, otro, la edad promedio fue de 18. (véase Figura 1).

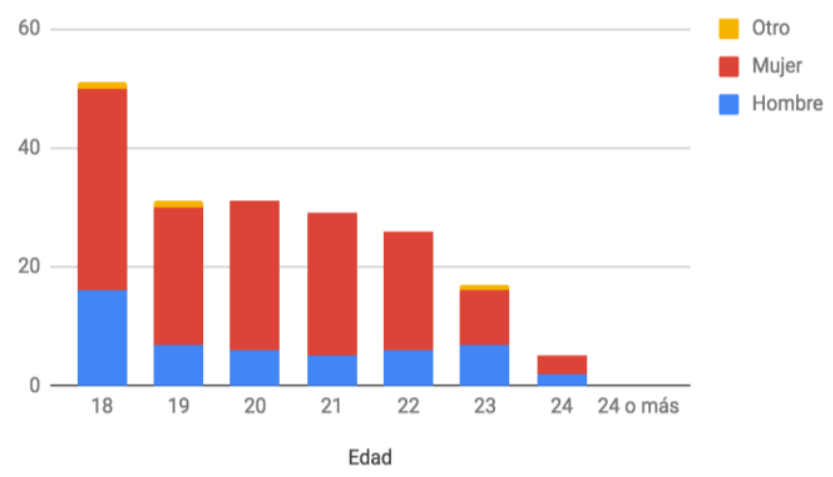

Figura 1. Género y edad de los usuarios de Instagram Fuente: Elaboración propia.

En cuanto al tipo de perfil que tienen las personas usuarias, un $28 \%$ de mujeres (40 personas) aseguraron tener un perfil de tipo «personal-privado»; mientras que, para los hombres, el mayor porcentaje $20 \%$ (10 personas) respondió que tenía de tipo «personal-público». El tipo «de negocios» fue el menos popular entre todos los géneros, con solamente un $4 \%$ (8 personas) entre ambos (véase Figura 2).

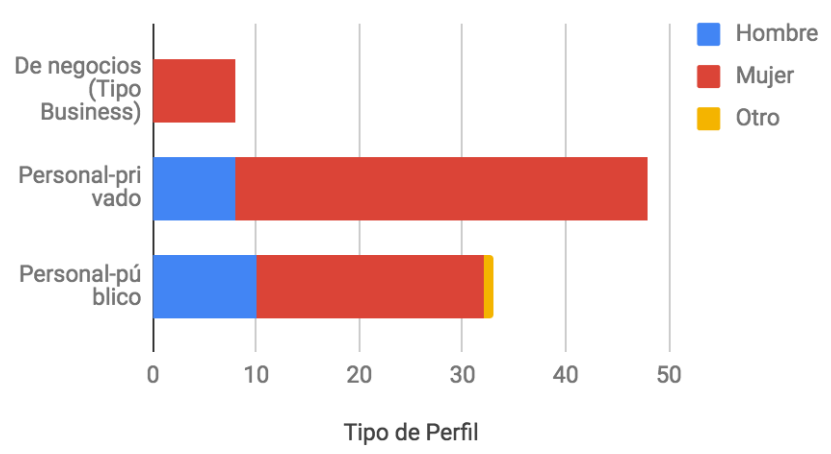

Figura 2. Tipo de perfil según género

Fuente: Elaboración propia 
Por otro lado, los jóvenes universitarios son consumidores activos en Instagram. Un 88\% (122 personas) de las mujeres y un 77\% (38 personas) de los hombres afirmó entrar «varias veces al día». Además, un 37\% (57 personas, 41 mujeres, 13 hombres y 3 otros) pasa por lo menos una hora al día dentro de la red social. No obstante, a pesar de frecuentar la red social de forma habitual, un $51 \%$ (45 personas) afirmó que publica solamente «una vez al mes», mientras que un $18 \%$ (16 personas) «más de una vez al mes».

En la Figura 3 observamos un alto uso por parte de los usuarios que publican en la aplicación varias veces al día o una vez al día. Las mujeres y otros son los usuarios más activos de la muestra encuestada publicando 1 vez al mes:

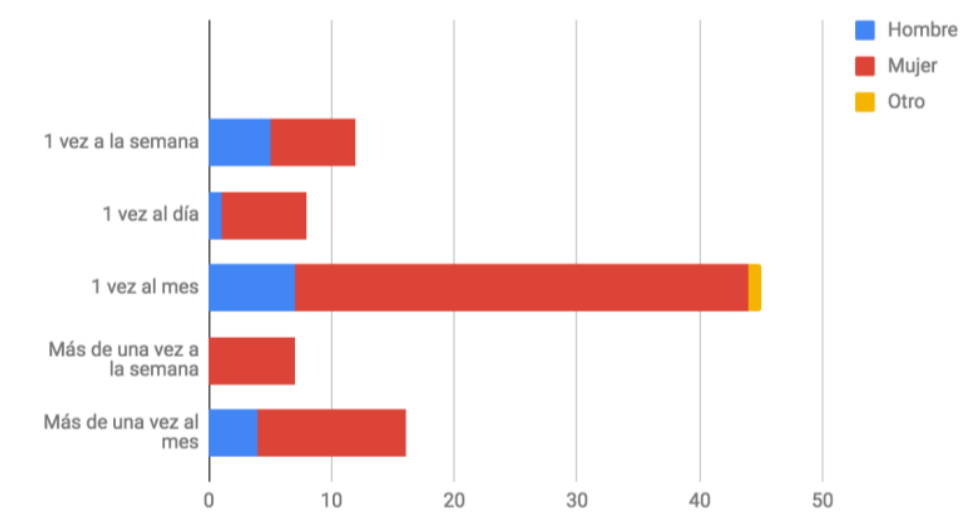

Figura 3. Frecuencia de publicación de contenido propio por sexo Fuente: Elaboración propia.

Aun cuando todos los géneros hacen referencia a postear contenido dentro de la plataforma, un 22\% (19 personas) afirmó que postear Historias es una actividad recurrente «más de una vez al día» y un $21 \%$ (18 personas) dijo que las publicaba «más de una vez a la semana»,. En su mayoría, las mujeres son quienes suelen utilizar más las Historias que los hombres. De las personas estudiantes, un 42\% (59 mujeres) y un 38\% (19 hombres) afirmaron seguir entre 200 y 500 perfiles registrados. Por otra parte, la segunda respuesta más popular entre las personas entrevistadas fue la de seguir entre 500 y 1000 perfiles, con un 26\% (36 mujeres) y un 18\% (9 hombres).

De igual forma, al preguntarles por el número de seguidores que tenían, la mayor cantidad de respuestas fue entre 250 y 500, representado por un $41 \%$ de mujeres (57 personas), por un $30 \%$ de hombres (15 personas) y otros (3 personas) entre 250 y 500 . La respuesta entre 500 y 1000 tomó el segundo lugar, con un 25\% (35 personas) de las mujeres y un $18 \%$ (9 personas) de los hombres. Solamente 13 mujeres y 4 hombres tienen más de 1000 (véase Figura 4). 


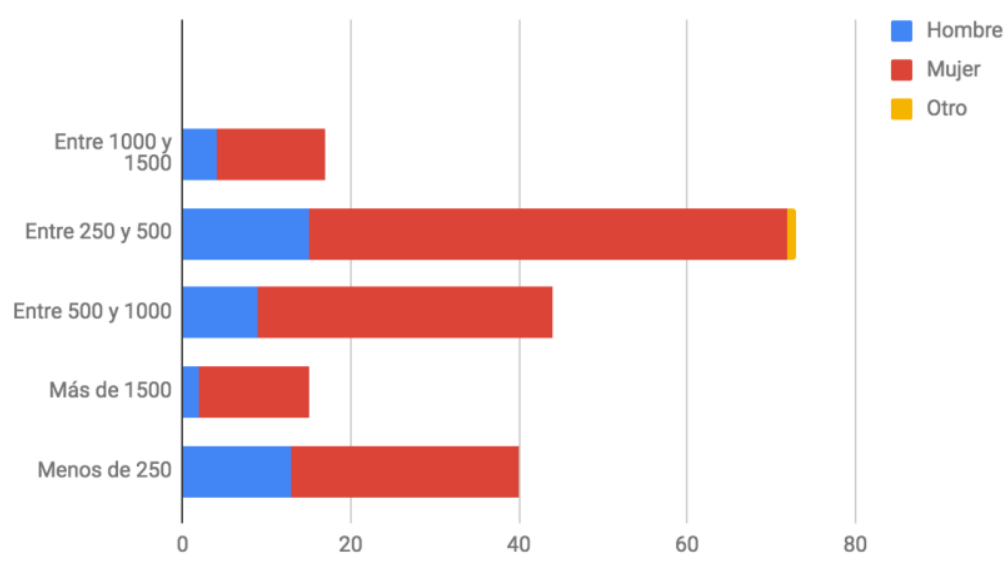

Figura 4. Cantidad de seguidores según sexo

Fuente: Elaboración propia.

Dentro de la encuesta, las personas participantes comentaron que usualmente no utilizan hashtags, pero quienes sí lo hacen suelen ser mujeres. Algunos de estos son \#TBT, \#Pride, \#Healthylifestyle, \#Fitness y \#Travel. Además, utilizan esta plataforma para estar en constante contacto con amigos, ya sea etiquetándolos en su contenido propio, chateando por el servicio de mensajería directa o bien, participando de forma activa al comentar sus posts. Otras de las opciones más mencionadas fueron el autoaprendizaje, el «tener vinculación con los medios de comunicación» y la revisión del feed o la fuente de noticias que se actualiza constantemente en estas redes sociales. Podemos indicar que las acciones de estas personas estudiantes desde su perfil y dentro de la red social son:

1. Revisión de la grilla de publicaciones en la página principal de Instagram.

2. Ver y publicar historias (Instagram Stories).

3. Ver y publicar contenido.

4. Observar las transmisiones en vivo (Livestreams).

5. Seguir marcas y darle me gusta (like) al contenido de las mismas.

6. Seguir usuarios tipo influencers (ver su estilo de vida y consumir contenido sobre los temas que publican).

La mayoría de las personas encuestadas, un $89 \%$ de las mujeres (124 personas) y un $81 \%$ (40 personas) de los hombres, afirmaron que, dentro de la red social, las personas deben apegarse a un estilo o forma de vida específico. Esto se aplica tanto para influencers como perfiles regulares.

Las personas encuestadas afirmaron, con un $86 \%$, ser seguidoras de organizaciones en Instagram. De estos, un 68\% (130 personas) corresponde a mujeres y un 17\% (33 personas) a hombres. No obstante, este porcentaje disminuyó al consultarles si seguían marcas comerciales 
o marcas personales en la plataforma. Solamente un 36\%, 59 mujeres, 15 hombres y 3 otros no , afirman ser seguidores de marcas comerciales (véase Figura 5).

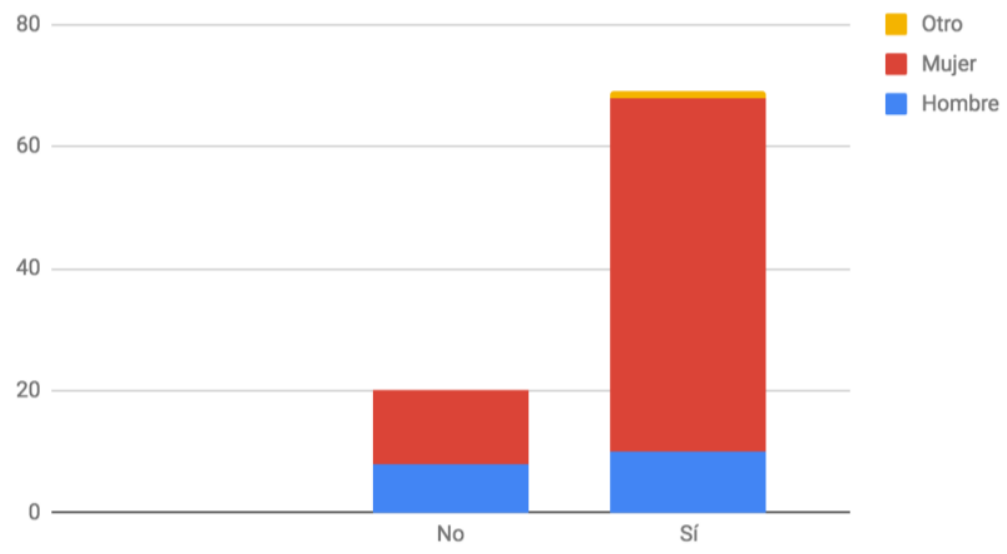

Figura 5. Seguimiento de organizaciones en Instagram Fuente: Elaboración propia.

Los tipos de marcas que siguen suelen ser variados. Las principales son marcas de moda con un $43 \%$ ( 82 personas), comidas y bebidas con un 35\% (67 personas), servicios de belleza con un $22 \%$ (42 personas) y tecnología con un $11 \%$ (22 personas). Un $32 \%$ asegura que le gusta ver actualizaciones de dichas marcas una vez a la semana. Podemos indicar que las interacciones del estudiantado con los perfiles de marcas en esta red social son:

1. Seguir marcas y darle me gusta al contenido de las mismas.

2. Tomar pantallazos de las publicaciones.

3. Comentar dentro de sus publicaciones.

4. Enviar su contenido por mensajería directa a alguien más.

Por otro lado, un 90\% de la población estudiantil afirmó que no se consideraba un usuario de tipo influenciador. Los encuestados comprenden el término influencer, la mayoría indica que lo conoce bien, sus características y su definición, por lo que indican en sus respuestas las razones por las cuales creen que no se consideran influencers. Según las personas entrevistadas, una persona influencers es alguien con muchos seguidores, que publica constantemente y a quien se le paga por hacerlo. Los motivos por los cuales no se consideran influencers, detallaron son por la falta de seguidores, porque realizan publicaciones de contenido poco constantes y que ninguna marca comercial los patrocina. Por otro lado, quienes sí se consideraron influencers, mencionaron que los temas que abordan son la promoción de vida saludable, publicación de fotografías de viajes y lugares, recomendaciones y consejos de bienestar personal. Sin embargo, varios hacían la aclaración de no contar con una gran cantidad de seguidores. 
Sobre algunos aspectos que definen a un influencers, los entrevistados menciona que estas personas se caracterizan por ser «atractivas», «carismáticas» y «simpáticas». Dentro de la encuesta, un $79 \%$ de las personas encuestadas afirmaron seguir perfiles de influencers en esta red social. En su mayoría, las mujeres son quienes tienden a seguir más este tipo de perfiles con un 64\% (122 personas) (véase Figura 6).

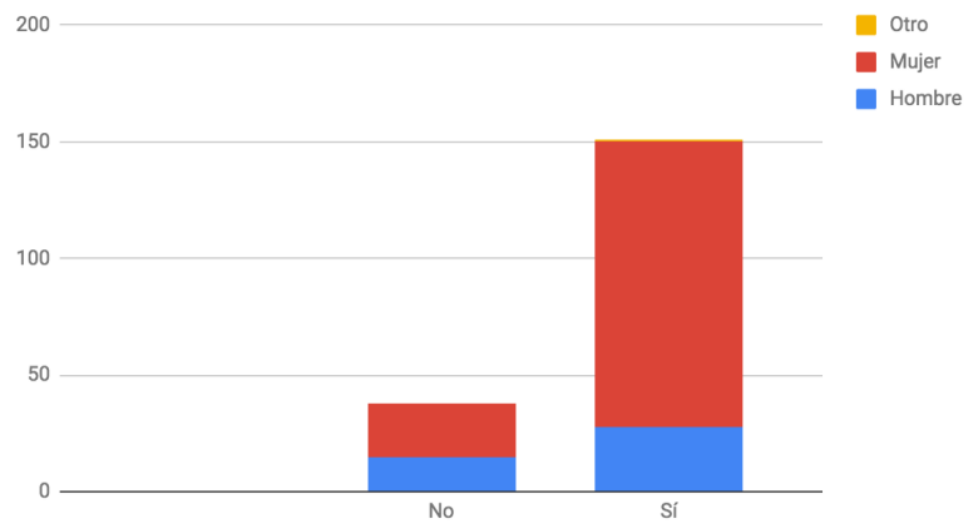

Figura 6. Seguimiento de influencers en Instagram Fuente: Elaboración propia.

Seguidamente cuando se les consultó que mencionaran a algún influencer, las personas encuestadas mencionaron influencers tanto nacionales como internacionales. Como se observa en la Tabla 1, se incluyen las personas influencers en el ámbito nacional; mientras que en la Tabla 2 se detallan las principales menciones a nivel internacional.

Tabla 1. Usuarios que fueron mencionados como influencers en Costa Rica

\begin{tabular}{|c|c|}
\hline Mención & Número de menciones \\
\hline Keylor Navas & 4 \\
\hline Nane Miller & 1 \\
\hline Johanna Solano & 1 \\
\hline Cristian Gamboa & 1 \\
\hline Joel Campbell & 1 \\
\hline Johanna Solano & 1 \\
\hline Kurt Dyer & 1 \\
\hline Señorita Abril & \\
\hline
\end{tabular}




\begin{tabular}{|c|c|}
\hline Leonora Jiménez & 1 \\
\hline Nancy Dobles & 1 \\
\hline Cata Freer & 1 \\
\hline Karina Ramos & 1 \\
\hline Javier Arce & 1 \\
\hline Kevin Poveda & 1 \\
\hline
\end{tabular}

Fuente: Elaboración propia.

Tabla 2. Usuarios que fueron mencionados como influencers en el mundo

\begin{tabular}{|c|c|}
\hline Mención & Número de menciones \\
\hline Jenner Kardashian & 22 \\
\hline Selena Gómez & 11 \\
\hline Justin Bieber & 7 \\
\hline Gigi Hadid & 7 \\
\hline Cara DeLevigne & 3 \\
\hline Emily Ratajkowski & Más de 50 menciones \\
\hline $\begin{array}{c}\text { Deportistas: Lionel Messi, Cristiano } \\
\text { Ronaldo, Antoine Griezzman, Neymar, } \\
\text { Zidane, Ronaldo. }\end{array}$ & Más de 40 menciones \\
\hline $\begin{array}{c}\text { Otros: Chloë Sevigny, Leonardo Dicaprio, } \\
\text { Jaden Smith, Lil Yachty, Jamie Oliver, } \\
\text { Björk, también perfiles de bandas. }\end{array}$ & \\
\hline
\end{tabular}

Fuente: Elaboración propia.

Como vemos los encuestados mencionan el nombre de celebridades cuando se les pregunta por influencers, es decir que el término influencers actúa como sinónimo del término celebridad, ya que cuando se les preguntó «¿Cuáles son los influencers con más seguidores en Costa Rica?», la mayoría aseguró no saber o no seguir a nadie dentro del país. Sin embargo, nombres como Keylor Navas, Nane Miller, Karina Campos, Alex Badilla, Choché Romano y 
Yiyo Alfaro, entre otros, fueron mencionados. Con respecto a la razón principal para seguirlos, un $48 \%$ de las personas estudiantes respondieron que ver su estilo de vida (49 mujeres y 16 hombres); y obtener información de temas específicos (55 mujeres y 10 hombres) se encontraban dentro de sus prioridades (véase Figura 7).

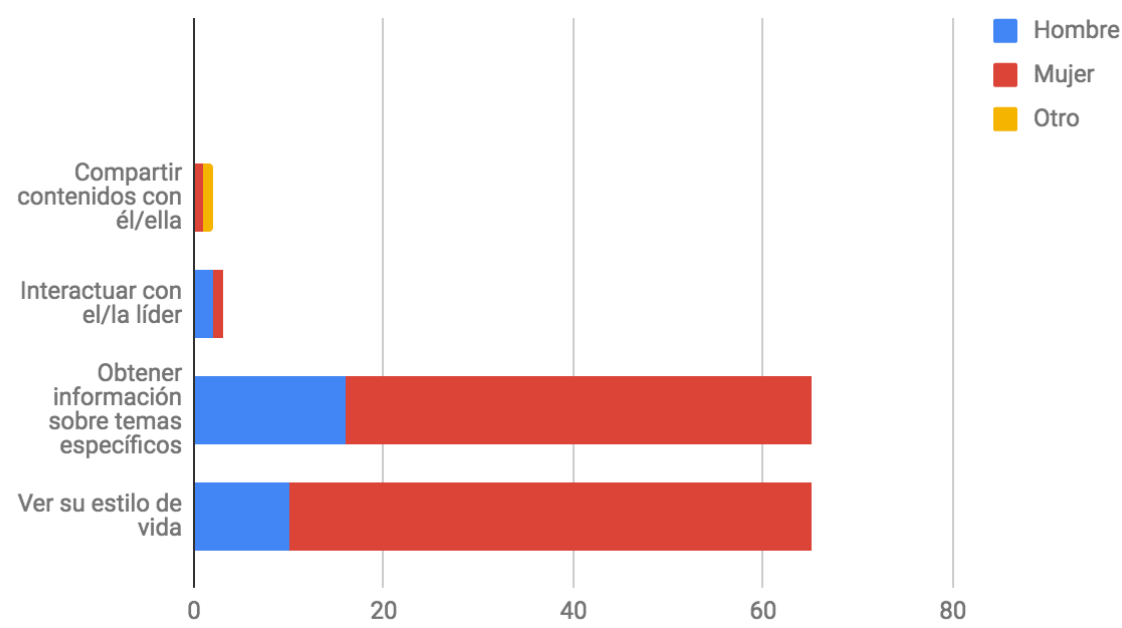

Figura 7. Razón principal para seguir influencers

Fuente: Elaboración propia.

Con respecto a los temas, los más recurrentes por parte de las personas identificadas con el género masculino, femenino y otros, fueron arte y cultura con un $72 \%$ (106 personas), con un $66 \%$ (98 personas) entretenimiento y con un 27\% (41 personas) política. Específicamente para las mujeres, los temas más populares que les generan interés en las publicaciones en Instagram, fueron el tema moda con un 29\% (43 personas), el tema bienestar con un 19\% (29 personas) y temas de belleza con $18 \%$ (27 personas). Entre los hombres destacaron como interés principal los temas relacionados con deportes con un $20 \%$ (30 personas) y temas de nutrición, con un $17 \%$ (25 personas).

Por otro lado, entre los contenidos que prefieren, las personas encuestadas aseguraron que buscan una combinación de Historias y publicaciones; es decir, un influencer que se mantenga activo de forma constante (véase Figura 8). 


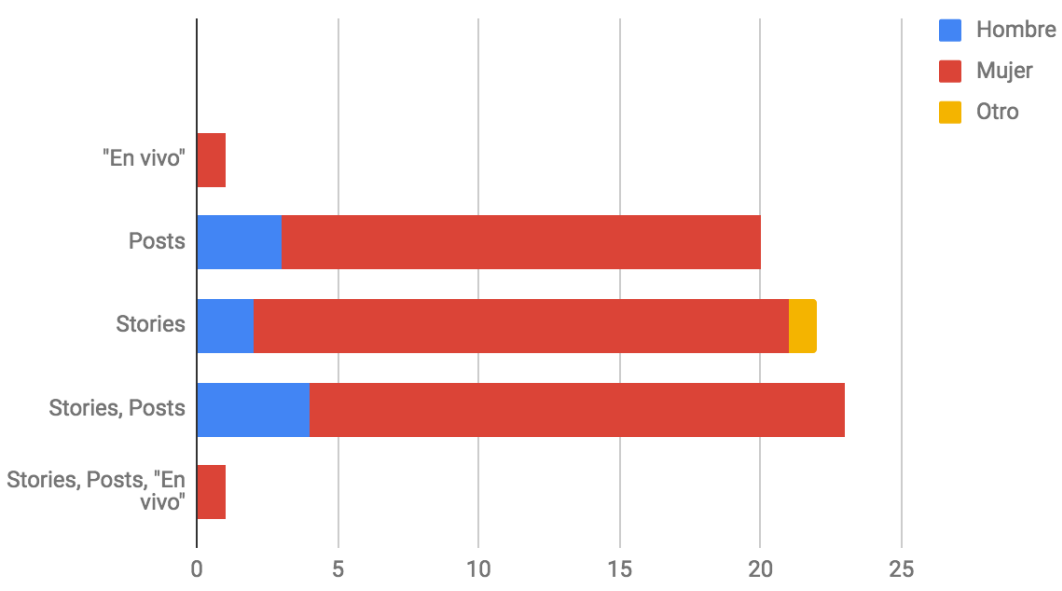

Figura 8. Contenido que prefiere de influencers

Fuente: Elaboración propia

En cuanto patrocinio de productos o servicios que realizan estas figuras dentro de la red social, la población estudiantil tiene una percepción mixta. Dentro de una pregunta abierta, se obtuvieron tanto comentarios positivos como negativos. Entre aquellas personas que rescataban esta labor, mencionaron frases como «genial cuando saben esconderlo, que no parezca publicidad invasiva», «que está bien mientras sea una opinión sincera» $\mathrm{y}$ «buena estrategia del marketing». Además, usaron adjetivos como «ingenioso».

No obstante, dentro de las personas encuestadas, frases como «no me parece que sea muy efectivo. Cuando no parece $100 \%$ natural, se siente forzado», «se saturan, o sea no creo que usen-coman todo lo que promocionan» $\mathrm{y}$ «aburrido» aparecían de forma constante. De hecho, un $51 \%$ (97 personas) aseguró sentirse molesto cuando un influencer promociona productos.

Bajo esta premisa, se puede inferir que existe un gusto por los productos que patrocinan los influencers, siempre y cuando este patrocinio no se encuentre construido de la misma forma que una publicación regular. El mismo puede verse mejorado, si efectivamente pareciera que las personas que anuncian el producto, lo hacen a manera de recomendación más que de promoción.

Siguiendo lo comentado, cuando se les preguntó si disfrutaban ver contenido promocional de marcas dentro de las stories, más de la mitad de las personas encuestadas ratificaron que esto les molestaba. Sin embargo, las mujeres en un 25\% (35 personas) y los hombres en un $18 \%$ ( 9 personas), mencionaron que, si tenían que ver, preferían que no se hiciera referencia al producto de forma directa, sino que se utilizaran recursos como los «detrás de escenas» para hablar del mismo (véase Figura 9). 


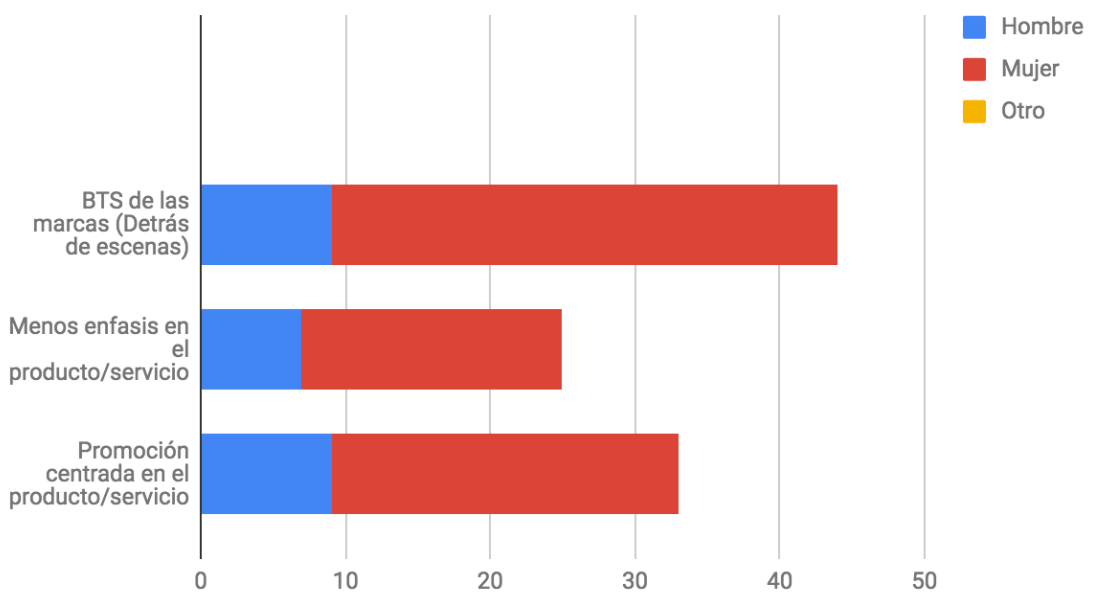

Figura 9. Contenidos promocionales en stories según sexo Fuente: Elaboración propia.

Al consultarles sobre la comunicación digital de estas marcas en Instagram, sucedió un fenómeno similar al señalado con los influencers. Si la publicidad era distribuida de forma excesiva, había un disgusto o rechazo generalizado por parte de las personas encuestadas. Un $37 \%$ aseguró que «una vez al día» era suficiente promoción, mientras que la segunda opción a la que se mostró más apego fue «una vez a la semana» con un $26 \%$. No obstante, a diferencia de la publicidad que realizan los influencers, dentro de estas no hubo molestia si no era discreta. Inclusive, señalaron que, en su mayoría, suele ser efectiva, puesto que se suelen mostrar los productos por medio de fotografías con una estética agradable.

Finalmente, un 49\% (93 personas) de quienes resolvieron la encuesta, afirmaron que la publicidad de Instagram puede volverse invasiva «a veces». Para un 63\% de las mujeres), un $55 \%$ de los hombres y $3 \%$ de otros, esto ocurre en especial cuando se utilizan dentro de las Historias. Para las personas encuestadas, la razón principal por la cual pueden percibir que una publicidad es invasiva, según el 64\% (89 personas) de las mujeres y el $46 \%$ ( 23 personas) para los hombres, recae en que el acercamiento que intenta ejecutar la marca se siente «impersonalartificial». Otro de los motivos que generan disgusto para el 22\% (31 personas) de las mujeres y $51 \%$ (14 personas) de los hombres, es que el producto o la publicidad se encuentra «en desacorde con la estética del feed». Un 10\% de las personas identificadas como hombre o mujer afirmó que, cuando una publicidad hace demasiado énfasis en el producto, tampoco es de su agrado (véase Figura 10). 


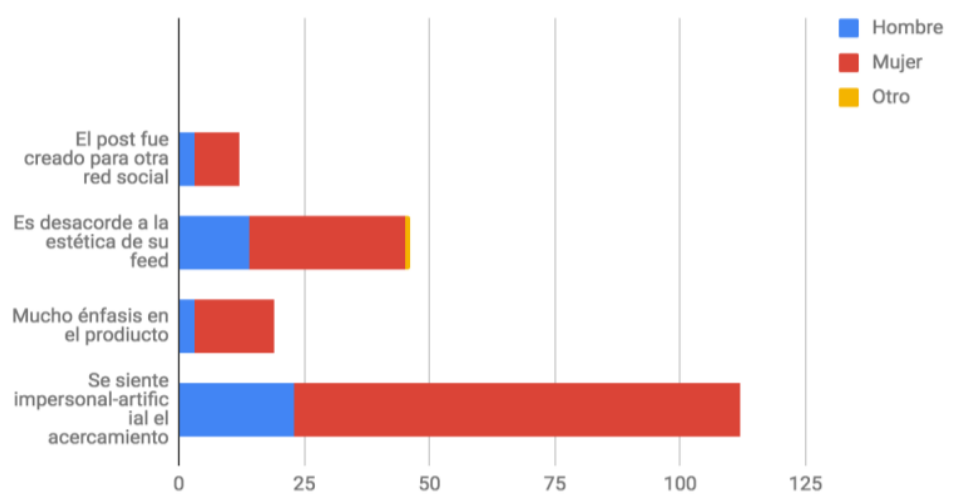

Figura 10. Razón de percepción invasiva según sexo Fuente: Elaboración propia.

Para finalizar, de acuerdo con el estudiantado, la principal forma de contrarrestar que una publicidad o promoción de un producto o servicio se sienta impersonal, es precisamente cuando el post se siente más personalizado. Por otra parte, otra de las soluciones mencionadas es que las publicaciones con contenidos publicitarios sigan un formato más acorde con la estética de la red con un $32 \%$ (45 personas) de las mujeres y un 28\% (14 personas) para los hombres.

\section{Conclusión}

Según los datos encontrados concluimos que existen una seria de formas y prácticas por medio de las cuales los millennials específicamente los jóvenes universitarios hacen uso de Instagram. Existen diferentes tipos de operación que explicaremos a continuación.

Primero, vemos que los jóvenes socializan y consumen información a través de Instagram. Si bien las mujeres son las que más comparten contenido como forma de expresión personal, esta red social potencia los procesos de comunicación entre millennials y marcas, por ser simple y fácil de usar y de un bajo costo.

Segundo, evidenciamos el surgimiento de un modelo económico entre personas usuarias, influencers y marcas en Instagram: un acercamiento al estudio de las prácticas en de interacción entre jóvenes, marcas e Instagram. Nos encontramos ante un usuario educado, exigente e interesado por la comunicación.

Los millennials interactúan activamente con las publicaciones de sus perfiles, de los perfiles de otros usuarios y de los perfiles de las marcas en Instagram. El nivel de interacción con otros perfiles está condicionado por la calidad de la información a nivel de temáticas y el uso que puedan darle ellos y ellas a esta información. Creemos que, entre los millennials, el uso de Instagram está impactando sus procesos de socialización con otros usuarios y con otro tipo de perfiles. Sin embargo, solamente el $36 \%$ de los entrevistados dice seguir una marca en Instagram. Recordemos que, para las empresas, las redes sociales son una poderosa fuente de datos obtenidas de las personas consumidoras. Es por esto que las marcas comerciales tendrían 
que plantearse como objetivo comprender las prácticas de la población joven en Instagram, y así conocer y las prácticas del espacio a partir de las operaciones que allí subyacen, así como las tácticas del uso de estas tecnologías.

Otro hallazgo del estudio fue evidenciar el papel de Instagram en los procesos de consumo de contenidos digitales por parte de las personas jóvenes. ¿Qué hacen las personas jóvenes en Instagram? Ver contenido de otras personas que usan la red social y de las marcas, influyendo así en sus compras por otras personas conocidas como influencers. Es decir, la población joven universitaria es una población consumidora activa, pero productora pasiva en Instagram. Su actividad principal es revisar la grilla de publicaciones, seguido de ver y publicar Historias. Las personas jóvenes siguen amando la inmediatez del contenido y su evaporación. Es un registro intermitente de sus contenidos. De ahí el éxito de la opción de las Historias de Instagram. Sobre este punto se recomienda, para futuras líneas de investigación, enfocar los esfuerzos en el análisis de tipos de contenido creados por los usuarios o uso de Instagram como herramienta colaborativa con otros usuarios.

Al analizar qué consumen los jóvenes en Instagram, se pueden tejer las perspectivas más relevantes acerca del impacto de esta red social en sus vidas, puesto que comprendemos de mejor manera qué hacen este tipo de usuarios en esta red social y cómo estos contenidos colaboran con este sentido de identificación y con la apropiación de la tecnología.

Finalmente, este estudio abre varias líneas de investigación que se deberían desarrollar en el futuro, algunas de las cuales pueden ser análisis de los discursos publicitarios de las marcas en Instagram, comprensión de los contenidos de los influencers en Instagram, la influencia de los perfiles de Instagram en otros ámbitos de la vida de los millennials y contenidos y formatos en Instagram.

Apoyo financiero: El siguiente estudio forma parte del proyecto «Los líderes de opinión digitales para la red de estudiantes universitarios» inscrito en el programa Prácticas de desarrollo y apropiación tecnológica del Centro de la Investigación de la Comunicación de la Universidad de Costa Rica.

\section{Referencias}

Andrés, Gonzalo. 2012. «Prácticas culturales en red: reflexión sobre la participación y producción de los internautas». Poliantea, 14: 97-110.

Brandwatch Analytics. (2016). 37 estadísticas de Instagram para 2016. Retrieved December 14, 2016. https://www.brandwatch.com/es/2016/05/37-estadisticas-de-instagram-2016/

Blommaert, Karel. 2016. The role of Instagram in creating brand awareness among the millennial skate culture of Cape Town.

Burgess, Jean. 2006. «Hearing ordinary voices». Continuum: Journal of Media \& Culture, 20 (2): 201-214. 
Carah, Nicholas. 2016. «Brands and Instagram: Point, tap, swipe, glance». Mobile Media \& Communication, 4(1): 69-84. DOI 10.1177/2050157915598180

Castells, Manuel. 2003. “La sociabilidad en Internet”, Lección Inaugural de Manuel Castells del programa de doctorado de Sociedad de la Información y el Conocimiento de la UOC. $\begin{array}{lllll}\text { Accedido } & \text { el } & 10 & \text { de } & \text { setiembre }\end{array}$ https://www.uoc.edu/web/cat/articles/castells/castellsmain7.html

de Certau, Michelle. 1990. L'invention du quotidien, tome 1: Arts de Faire. UGE: Paris.

Endres, Hannah. 2013. Shreddz a Clothing Brand: Creating a Marketing Plan Through Social Media and Traditional Marketing Research. Estados Unidos: California Polytechnic State University.

Eunji, Lee, Jung-Ah Lee, Moon, Jang Ho Moon y Yongjun Sung. 2015. «Pictures Speak Louder than Words: Motivations for Using Instagram». Cyberpsychology, Behavior, and Social Networking, 18 (9): 552-556. https://doi.org/10.1089/cyber.2015.0157

Hootsuite. 2017. «Una lista completa de estadísticas de Instagram». https://blog.hootsuite.com/es/lista-completa-de-estadisticas-de-instagram/

Instagram «700 millones». 2017. Instagram, 26 de abril de 2017. https://instagrampress.com/blog/2017/04/26/700-million/ Recuperado 4 de mayo de 2018.

J.D. Power and Associates. 2013. «Social Media Benchmark Study» https://www.jdpower.com/business/press-releases/2013-social-media-benchmark-study

Kotler, Philip y Keller, Kevin. 2012. Dirección de Marketing. Décimo cuarta ed. México: Pearson.

Macon, June. 2017. «An Examination of Users and the Commodification of Instagram Posts». Tesis de maestría, Universidad del Estado de Colorado. https://papers.ssrn.com/sol3/papers.cfm?abstract_id=2944502

Manovich, Lev. 2008. "Introduction to Info-Aesthetics". Reflexiones en el blog Manovich.Net http://manovich.net/index.php/projects/introduction-to-info-aesthetics

Red506 2017-VII Edición-El Financiero. 2017. 10 de octubre de 2018. https://www.elfinancierocr.com/gnfactory/ELF/GNF/2017/06/30/0001/Red506-VIIEdicion-Financiero_19_1204269562.html

Siles, Ignacio. 2004. «Sobre el uso de las tecnologías en la sociedad. Tres perspectivas teóricas para el estudio de las tecnologías de la comunicación». Revista Reflexiones, 83(2): 7382.

UNIMER. s.f. «Costa Rica cuenta con su propio estudio de generaciones». https://blog.unimercentroamerica.com/costa-rica-cuenta-con-su-propio-estudio-degeneraciones Recuperado 4 de mayo de 2018. 